\title{
Contrôle de la transition métaphase-anaphase
}

Une cellule, pour se diviser, doit d'une part répliquer son matériel génétique et d'autre part dupliquer son centre de nucléation des microtubules : le centrosome. Cette duplication est indispensable à l'organisation du fuseau bipolaire de microtubules qui constituera l'ossature sur laquelle s'exerceront les forces nécessaires à la séparation des chromosomes en deux lots équivalents lors de la mitose. Durant cette phase, les chromosomes doivent, en premier lieu, acquérir un attachement bipolaire, c'est-à-dire que les chromatides sœurs de chaque chromosome bivalent doivent s'associer aux microtubules émanant des pôles opposés du fuseau par l'intermédiaire des kinétochores (structure protéique assemblée sur l'ADN centromérique). Une fois la bi-orientation acquise, les chromosomes bivalents migrent vers la plaque équatoriale du fuseau, c'est la phase de congression. Lorsque tous les chromosomes sont alignés sur la plaque équatoriale, la cellule est en métaphase. A la transition métaphase-anaphase les chromatides sœurs de tous les chromosomes se séparent simultanément et migrent vers les pôles opposés du fuseau. La capture et la bi-orientation des chromosomes est un phénomène stochastique, la durée de cette phase ne peut donc être prédéterminée. L'anaphase ne se déclenchant qu'après l'alignement du dernier chromosome sur la plaque métaphasique (dans le cas contraire, les risques d'aneuploïdie seraient énormes), il existe nécessairement un mécanisme de couplage entre l'alignement des chromosomes et le début de l'anaphase. Ce mécanisme est aussi appelé point de contrôle mitotique (figure 1).

L'entrée en anaphase est contrôlée par la dégradation de protéines régulatrices clefs, comme par exemple les sécurines, dont la destruction est requise pour la séparation des chromatides sours [1]. La spécification des protéines à dégrader est assurée par la fonction de l'anaphase promoting complex (APC). L'APC est une E3 ligase, qui, en conjonction avec un activateur, Cdc20/ Fizzy, ubiquitinyle les protéines cibles permettant ainsi leur reconnaissance par le protéasome et leur dégradation. Bien que I'on ne connaisse pas, à l'heure actuelle, les mécanismes intimes qui couplent I'alignement des chromosomes avec le déclenchement de I'anaphase, il semble évident que les kinétochores non capturés par les microtubules sont le lieu d'émission d'un signal empêchant la mise en route de la machinerie de dégradation dépendante de I'APC Fizzy. En effet, la destruction au laser du dernier kinétochore libre, dans une cellule où tous les autres chromosomes

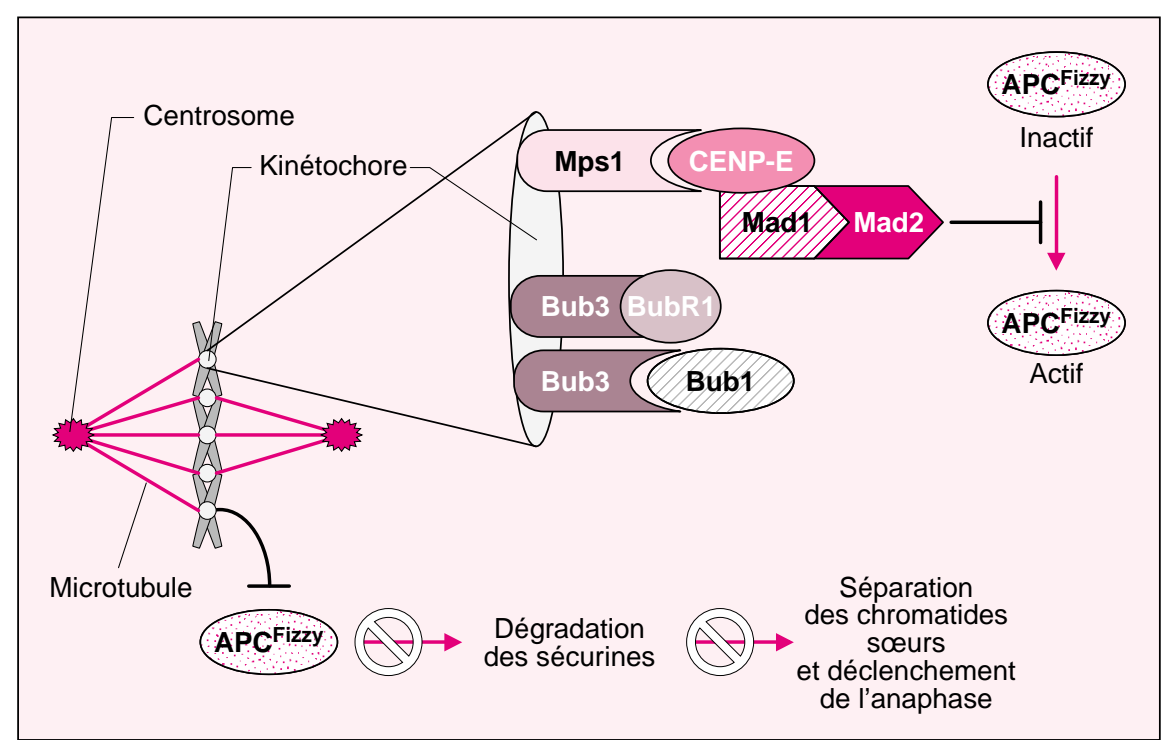

Figure 1. Modèle du mécanisme de couplage entre métaphase et anaphase. Le point de contrôle du fuseau mitotique retarde le début de l'anaphase jusqu'à ce que tous les chromosomes acquièrent un attachement bipolaire stable avec les microtubules du fuseau mitotique. Mpsl est nécessaire pour recruter CENP-E sur les kinétochores, lui-même étant requis pour le recrutement de Mad1 et Mad2, nécessaire à l'émission d'un signal d'attente bloquant l'activité du complexe APC Fizzy et le déclenchement de l'anaphase (les interactions représentées entre Mpsl et CENP-E, CENP-E et Mad1 reflètent l'ordre hiérarchique de recrutement et non pas une interaction directe). L'activité de la kinase Mpsl est essentielle à l'expression de ce point de contrôle. Toutefois, le ou les substrats de cette kinase restent à déterminer. Les protéines CENP-E, BubR1, Bub1 et Bub3 sont des candidats potentiels. 
ted by benomyl), conduit à une défaillance de l'arrêt du cycle cellulaire en mitose sous l'influence du benomyl et aboutit à la mort cellulaire. Par la suite le gène M psl initialement découvert pour son caractère essentiel dans la duplication du spindle pole body (centre organisateur des microtubules chez la levure) a également été impliqué dans le point de contrôle mitotique. Des homologues de ces gènes ont été caractérisés chez les vertébrés (avec une exception pour Mad3 dont I'orthologue est un hybride entre Mad3 et Bub1 appelé BubR1) suggérant que la base moléculaire de ce mécanisme est conservée au cours de l'évolution. Par ailleurs, la présence des protéines $M$ ad et Bub sur les kinétochores, et préférentiellement sur les kinétochores libres, est en adéquation avec la notion que ces derniers produisent un signal inhibant l'entrée en anaphase. Les démonstrations, d'une part que Mad2 interagit physiquement avec APC Fizzy et inhibe son activité, et d'autre part, que I'interaction de $M$ ad 2 avec les kinétochores non alignés est hautement dynamique (renouvellement de la moitié des molécules en 24 secondes) [2], ont conduit à une hypothèse simple sur la nature de ce signal : les kinétochores libres seraient des usines à fabriquer et à relarguer une forme transitoire de Mad2 capable d'inhiber $A P C^{\text {Fizzy }}$ et de retarder le déclenchement de l'anaphase. Sont également en accord avec cette hypothèse, des études menées dans notre laboratoire sur le modèle des extraits cyclants d'œufs de xénope. Ces extraits, Iorsqu'ils sont supplémentés en chromosomes provenant de têtes spermatiques, sont capables d'alterner des phases de réplication et des phases mitotiques comme l'auraient fait les œufs dont ils sont issus. Ils sont aussi capables de s'arrêter en phase mitotique en réponse à la présence de kinétochores libres obtenus par adjonction de nocodazole. L'avantage de ce modèle est qu'il permet facilement d'éliminer ou de complémenter l'extrait de la protéine étudiée. Dans ces conditions, la présence de Mad2 sur les kinétochores dépend de la présence de nocodazole et par ordre hiérarchique dépend de la présence de $M$ ad1, de la chromokinésine CENP-E et de l'orthologue chez les vertébrés de la protéine kinase Mps1 [3] . La localisation sur les kinétochores de Madlet Mad2 dépend également de la présence du complexe Bub1-Bub3 dans lequel Bubl est aussi une protéine kinase. L'ordre hiérarchique entre Bub1-Bub3 et Mps1 reste encore à déterminer. La déplétion, dans l'extrait de xénope, de chacune de ces protéines conduit à l'absence de Mad2 sur les kinétochores libres et à la perte du bloc mitotique.

Le modèle du kinétochore libre comme seule source de facteur inhibiteur de l'anaphase apparaît néanmoins contrarié par la démonstration qu'un complexe formé de protéines contenant BubR1, Bub3, Cdc20/ Fizzy et Mad2 possède une activité inhibitrice sur I'APC [4]. Ce facteur préexiste dans des cellules en interphase, alors que les kinétochores ne sont pas encore matures, ce qui suggère qu'un mécanisme constitutif de répression de I'APC Fizzy existe. Par ailleurs, des expériences de fusion cellulaire ont montré que si deux fuseaux mitotiques sont présents dans la même cellule, il suffit que l'un d'eux atteigne la métaphase pour que les deux déclenchent I'anaphase simultanément, même si l'autre présente toujours un chromosome en retard [5]. Cette observation suggère plutôt que le signal émis par les kinétochores libres prévient localement l'émission d'un autre signal qui, lui, est envahissant et positif sur le déclenchement de l'anaphase.

Bien qu'encore très schématiques, nos connaissances du mécanisme de couplage entre métaphase et anaphase ne cessent de progresser. Elles mettent en valeur l'importance de ce mécanisme dans le développement d'un organisme et il apparaît de plus en plus que ses défaillances sont, pour une bonne part, à l'origine de l'instabilité chromosomique observée dans les cellules de la plupart des tumeurs, contribuant ainsi probablement au processus de tumorigenèse lui-même.

1. Abrieu A, Dorée M. La cohésion des chromatides sœurs et sa régulation au cours du cycle cellulaire. M ed Sci 2001; 17: 353-4.

2. Howell BJ, Hoffman DB, Fang G, Murray AW, Salmon ED. Visualization of Mad2 dynamics at kinetochores, along spindle fibers, and at spindle poles in living cells. J Cell Biol 2000; 18: 308-19. 3. Abrieu A, Magnaghi-Jaulin L, Kahana JA, Peter $M$, Castro A, Vigneron S, Lorca T, Cleveland DW, Labbe JC. M psl is a kinetochore-associated kinase essential for the vertebrate mitotic checkpoint. Cell 2001; 12: 2660-71.

4. Sudakin V, Chan GK, Yen TJ. Checkpoint inhibition of the APC/ C in $\mathrm{HeL}$ a cells is mediated by a complex of BUBR1, BUB3, CDC20, and MAD2. J Cell Biol 2001; 154: 925-36.

5. Rieder CL, Khodjakov A, Paliulis LV, Fortier TM, Cole RW, Sluder G. Mitosis in vertebrate somatic cells with two spindles: implications for the metaphase/ anaphase transition checkpoint and cleavage. Proc Natl Acad Sci USA 1997; 94 : 5107-12.

\section{Jean-C laude Labbé Thierry Lorca}

Cen tre de recherche de biochimie macromoléculaire, Cnrs UPR 1086, 1919, route de $M$ ende, 34293 M ontpellier Cedex 5, France.

\section{Journées Internationales Henri-Pierre-Klotz d'Endocrinologie Clinique 23-24 mai 2002}

Société Française d'Endocrinologie

Une décennie de progrès en thyroïdologie

Renseignements : Dr G. Copinschi,

Laboratoire de médecine expérimentale, Université Libre de Bruxelles, CP 618

808, route de Lennik, B-1070 Bruxelles, Belgique 